\section{Restoring Hope and Dignity CVT Manual}

Published by CVT (ISBN: 978-0975978948)i

\section{Marianne C Kastrup, MD, PhD}

The Restoring Hope and Dignity CVT Manual was developed by the Center for Victims of Torture in Minnesota and is meant for group counselling. It was made possible due to financial support from different US Agencies and the US Department of State's Bureau of Population.

The manual focuses on group counselling interventions and is based upon 15 years of experience from around the world, including Guinea, Sierra Leone, Liberia, the Democratic Republic of Congo, Kenya, Ethiopia, Jordan, and training and capacity building in Uganda, Bosnia, Cambodia, Sri Lanka, Cameroon, South Africa, Moldova and Lebanon. The model described has evolved gradually over this time based upon the experience and feedback from the involved counsellors and trainers and importantly from the very large group of clients.

As can be seen from the list of countries where the model has been used, it is intended primarily for humanitarian or low-resource settings where war and human rights violations have left the population in extreme distress and with a concomitant low level of functioning. The intention is that the interventions described should be delivered by trained counsellors in local settings. It

${ }^{\mathrm{i}}$ www.cvt.org 2016 needs to be emphasied that the model may be implemented even if counsellors have limited previous training in mental health issues, but it is important that they receive ongoing supervision and training. Counsellors with previous experience and with an academic background within a given local context may of course also provide the intervention.

To overcome the consequences of atrocity, a 10 session counselling model has been developed. Elements are taken from different theories such as cognitive behavioural theory, narrative exposure therapy, somatic psychology, interpersonal therapy and from different evidence-based trauma interventions. The model pays particular tribute to Judith Herman's tri-phase stages of trauma recovery. In an initial phase of safety and stabilization, focus is directed towards providing a safe physical environment for counselling. The clients are taught grounding techniques, and receive psycho-education related to trauma and its effects, but are also helped to understand the connection of thoughts and feelings. The aim is to support the clients in achieving greater emotional security.

In the second phase, the focus is on remembrance and mourning. The idea is that clients should come to terms with the traumata they have experienced and the impact on their life through processing traumatic memories. A mourning process will take place over previous loss and clients are supported in working with difficult feelings that have arisen as a consequence of the traumatic experiences. Finally, in the third reconnection phase, clients will begin to look towards the future, set goals, and hopefully be able to reconnect with themselves and their community, as well as finding new meaning and purpose in life.

The manual also provides a comprehen- 
sive, easy-to-grasp overview of the practical dimensions of the intervention, starting with the assessment of whether the client may benefit from group-counselling at all, and whether any adjustments are needed. It is in this initial phase that the first steps of the therapeutic relationship are taken, in an atmosphere of respect and empathy.

The following step comprises the treatment planning. According to the client's needs, the plan may involve several dimensions including individual or family treatment, medical and psychiatric care, physiotherapy, or social work. The overall idea is to have a holistic perspective and ensure that no problem is neglected. Counselling in a group may be very beneficial for some clients as they get the feeling that they are not alone, and that many others may experience similar problems. The model emphasizes that group counselling needs live supervision, with an emphasis on self-care and that the manual should be used combined with supervision.

Ethical and safety aspects - emotional as well as physical - are important parts of the model. Any processing of traumatic experiences needs an environment of emotional safety where clients are informed about confidentiality and professional boundaries. But the physical part should not be neglected, and it recognizes the importance of a warm, friendly environment where it is possible to feel at ease.

The model describes a structure where each session follows a fixed pattern. All sessions start with an opening - a kind of check-in to both evaluate how clients are doing as well as a kind of recap from the last session. In doing so, it is intended that clients stay focused on what were important issues at the previous session, what has happened since then, and what should be the theme for the next session. From there, the session then moves to the working part, where clients are taught new coping skills and time may be spent on remembrance and mourning. Finally, the session ends with a closing exercise helping the clients to integrate the lesson learned and prepare to move on - all carried out in 90 minutes.

To implement this requires that the counsellor is well-prepared and stays focused, and the manual provides ample information that is valuable for the counsellor to achieve this.

The manual outlines the 10 sessions: Introduction and Creating a Safe Space (1); Using our Strengths and Resources to Help Us Cope (2); Connecting our Minds and Bodies (3); River of Life: Honoring Our Life Story (4); Working Through a Traumatic Memory (5\&6); Addressing Multiple Losses and Reclaiming Goodness (7); Living with the Loss of a Loved One (8); Reconnecting to Self, Community and the Future (9) and Integrating and Saying Goodbye (10). Rich practical advice for each session, which is both easy to use and very illustrative, is also included, as are useful annexes, namely, (a) assessment training material, which is a detailed explanation of the CVT's assessment process; and, (b) handouts for closing practice.

The manual's strength lies in it being based on extensive experience in a number of settings involving clients from different cultural backgrounds and types of adversity. Furthermore, it is easy to read, also for non-academic staff; it is generously illustrated and uses a language that is full of respect and understanding for the variety of settings in which counsellors have to work. I recommend that it should be widely used and that there should be a continuous interaction between users of the manual and those responsible for its publication in order to monitor any limitations and shortcomings to ensure its ongoing improvement. 